\title{
Faut-il déclarer le «niveau 2 épidémie » face à la progression du nombre de robots chirurgicaux?
}

\author{
G. Meurette \\ (C) Lavoisier SAS 2020
}

Le 10 janvier 2020 était publié dans JAMA un article relatant la progression des actes par voie robot-assistée dans l'état du Michigan aux États-Unis entre 2012 et 2018 [1]. Cet article épidémiologique montre une augmentation importante du recours à l'assistance robotique pour les interventions de chirurgie viscérale et digestive. Le taux de recours au robot est passé de 1,8 à 15,3\% : multiplié par 8 ! «C'est un grand succès, une progression irrémédiable, témoin s'il en faut d'un progrès chirurgical majeur. Peut-être même s'agit-il d'une révolution » comme d'aucuns se complaisent à le relayer pour culpabiliser ceux qui n'ont pas encore choisi de s'équiper.

Après lecture attentive du manuscrit, $\mathrm{j}$ 'ai ressenti une grande frustration, car il ne répond pas à la question qui m'est immédiatement venue à l'esprit en reposant l'article sur le bureau : mais pourquoi cette progression?

En effet, à ce jour, les seules preuves factuelles du recours à l'assistance robotique sont :

- son surcoût (reste à charge pour le patient ou pour l'institution) dans quasiment toutes les indications évaluées dans la littérature ;

- une absence de supériorité en termes de marges de résection en chirurgie carcinologique, de conversion en chirurgie ouverte, de diminution des douleurs postopératoires ;

- une diminution à la marge et de façon inconstante dans certaines publications de la durée d'hospitalisation et des pertes sanguines.

Les conclusions des articles sont chaque fois identiques: "le robot n'est pas inférieur à la laparoscopie, c'est plus cher, mais on voit mieux ». Difficile de trouver dans ces arguments une explication rationnelle à la progression de l'approche robotique. On pourrait même raisonnablement penser qu'avec cette littérature en poche pour monter un dossier à présenter au directeur de la commission investissement de son hôpital (c'est fou le nombre de directeurs...), on a peu

\section{G. Meurette $(\bowtie)$}

Clinique de chirurgie digestive et endocrinienne, CHU de Nantes, Hôtel-Dieu, 1, place Alexis-Ricordeau, F-44000 Nantes, France e-mail : gmeurette@hotmail.com de chance de le convaincre... En tout cas, il n'y a rien de révolutionnaire.

Mais il y a encore plus surprenant : à la lecture du détail des interventions pour lesquelles l'assistance robotique est utilisée, la plus forte progression est... La hernie inguinale ! Eh oui, à en croire les chiffres, un tiers des hernies inguinales en 2018 sont opérées avec une assistance robotique. Les bras m'en tombent, ma plume sèche. Normalement, le but d'investir dans la technologie innovante en chirurgie est de dépasser nos limites là où elles sont. Pour les chirurgiens colorectaux, c'est indiscutablement la dissection du pelvis profond dans les cancers du bas rectum, dans les prolapsus complexes récidivants et douloureux, les patients multiopérés. Mais un lecteur éclairé de Colon Rectum peut-il m'expliquer ce qui justifie l'utilisation du robot dans un tiers des hernies inguinales? «Je n'ai pourtant aucun a priori sur la recherche dans le domaine de la chirurgie pariétale : qu'on mobilise des moyens pour mettre au point des prothèses qui ne s'infectent pas, qui se résorbent (ou pas), qui ne donnent pas de douleurs, mais que vient faire le robot dans cette affaire?»

Parmi les hypothèses avancées pour expliquer cette progression, on pourrait citer " l'excuse » de la courbe d'apprentissage, les chirurgiens réservant les premiers cas de chirurgie robotique à des cas simples et peu risqués. Cette attitude est légitime et peut expliquer en partie certains recours discutables au robot ; mais pas dans des proportions citées par ce papier.

L'autre hypothèse à avancer est moins glorieuse, moins médicale, mais bien à l'esprit de chacun de nous : c'est une politique incitative auprès des chirurgiens. Sans aller jusqu'au complot politico-industriel (je regarde trop les séries « Netflix » et lis trop de romans complotistes !), on comprend bien qu'après avoir investi dans du matériel (très) coûteux nos « financeurs » aient envie de voir leur tableau prévisionnel d'amortissement scrupuleusement respecté. «Puisque nous avons la machine, maintenant il va falloir l'utiliser ! ». En écrivant ces mots que je pèse, j'ai conscience d'être excessif. Les chirurgiens (dans le Michigan aussi) sont intelligents, ils ont gardé leur libre arbitre, et nous revendiquons tous une totale liberté dans nos indications 
opératoires, c'est une certitude. Mais reconnaissons aussi que les conditions d'exercice ont beaucoup changé en 20 ans. Nous sommes aujourd'hui dépendants de plateaux techniques et de structures qui bien souvent imposent plus qu'elles ne disposent. Ces contraintes influencent nécessairement notre pratique. Il est donc important parfois de remettre en question la pertinence de nos choix comme le fait assez justement cet article.

Il nous faut avoir un sursaut de lucidité et définir ensemble précisément les règles d'utilisation des instruments coûteux. Le robot peut être un progrès, mais il faut absolument :

- utiliser ces machines dans des situations où elles sont supposées apporter un bénéfice avant tout ;

- évaluer dans le cadre d'un registre exhaustif (et peut-être contraignant) avec des paramètres standardisés les bénéfices attendus (mais aussi la sécurité des patients) et une évaluation médicoéconomique fiable.

Cette publication scientifique concerne un État des ÉtatsUnis, et provient des données d'un registre assuranciel dont on peut aussi douter du caractère exhaustif. Elle n'est donc qu'un reflet plus ou moins précis de l'activité.

Où en sommes-nous en France ? Personne ne le sait réellement, et ce constat doit changer. Il y a des initiatives positives, comme les différents essais prospectifs randomisés obtenus par des financements institutionnels qui sont en cours dans différentes spécialités. La caisse d'assurance maladie a également créée un code d'extension documentaire que l'on peut apposer à l'acte lorsque l'intervention a été effectuée au robot. Cet indicateur n'est pas valorisé actuellement, mais il a pour but de dresser des statistiques exhaustives du recours au robot sur le territoire (s'il n'est pas valorisé le sera-t-il vraiment ?). Des registres se créent comme le GROG qui est un registre de suivi de patients opérés d'un cancer du rectum au robot. D'autres sont probablement en cours dans d'autres spécialités.

Si nous croyons à l'avenir de la chirurgie robotique dans sa capacité d'améliorer nos gestes, de préciser les dissections, d'apporter de l'intelligence artificielle à nos décisions opératoires, il faut absolument que nous respections une charte d'utilisation fidèle à notre rigueur scientifique et libre de toute autre influence. C'est plus difficile qu'avant certes, mais indispensable pour préparer l'avenir de ceux qui nous succéderont et qui nous opéreront !

Pour conclure, la lecture de cet article m'amène à la conviction que nous devrions mieux établir les règles d'utilisation du robot, et que nous ayons plus de transparence. Pour répondre à la question, j'élèverai le niveau de vigilance pour éviter l'épidémie...

Professeur Guillaume Meurette

Chirurgien utilisateur plurihebdomadaire du robot dans des indications précises et en cours d'évaluation.

Liens d'intérêts : l'auteur déclare ne pas avoir de liens d'intérêts.

\section{Référence}

1. Sheetz KH, Claflin J, Dimick JB (2020) Trends in the adoption of robotic surgery for common surgical procedures. JAMA Network Open 3:e1918911 